Pacific Journal of Mathematics

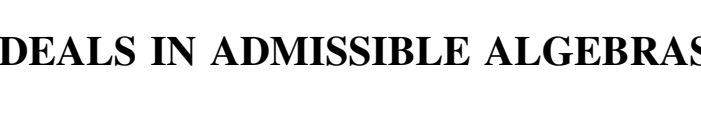




\title{
IDEALS IN ADMISSIBLE ALGEBRAS
}

\author{
EARL J. TAFT
}

The notion of admissible algebra has been introduced by Koecher. They are commutative algebras whose enveloping Lie algebra (of multiplications) splits into the direct sum of an even and an odd part. It will be shown here that the class of admissible algebras cannot be defined by (nonassociative) polynomial identities. This is done by exhibiting an admissible algebra which possesses a homomorphic image which is not admissible. The main tool is the relationship between the admissibility of a homomorphic image of an admissible algebra $A$, a symmetry property of a certain ideal of the enveloping Lie algebra of $A$ formed from the kernel of the homomorphism, and the ideal structure of an algebra constructed by Koecher from $A$.

Let $A$ be a commutative algebra over a field $F$ of characteristic not two. We do not assume that $A$ is associative, nor that $A$ has a unit element 1 . For $a$ in $A$, let $L(a)$ denote multiplication by $a$ in $A$, i.e., $L(a) x=a x$ for $x$ in $A$. Let $L(A)$ denote the vector space of linear mappings $L(a), a$ in $A$. Let $H(A)$ be the Lie algebra generated by $\mathrm{L}(A) . \quad H(A)=H^{-}(A)+H^{+}(A)$, where $H^{-}(A)$ is the space spanned by commutator products of an odd number of elements of $L(A)$, and $H^{+}(A)$ is the space spanned by commutator products of an even number of elements of $L(A)$. The commutator [T, $U$ ] of $T$ and $U$ is $T U-U T$. If $1 \in A$, then the sum $L(A)+[L(A), L(A)]$ is direct, since commutators vanish on 1 . We call $A$ admissible, [1], if the identity mapping of $L(A)$ extends to an antiautomorphism $T \rightarrow T^{*}$ of $H(A)$. This means that $T^{*}=T$ for $T \in H^{-}(A)$, and $T^{*}=-T$ for $T \in H^{+}(A)$, so that $H^{-}(A) \cap H^{+}(A)=0$. Conversely, if $H^{-}(A) \cap H^{+}(A)=0$, then the map $(T+U)^{*}=T-U$ for $T \in H^{-}(A), H \in H^{+}(A)$ shows that $A$ is admissible.

Let $A$ be admissible. We may then form the algebra $\mathbb{2}(A)=$ $H(A) \oplus A \oplus \bar{A}$, where $\bar{A}$ is a vector space copy of $A$. The multiplication in $\mathcal{L}(A)$ is given by $\left[\left(T_{1}, a_{1}, \bar{b}_{1}\right),\left(T_{2}, a_{2}, \bar{b}_{2}\right)\right]=(T, a, \bar{b})$, where $T=$ $\left[T_{1}, T_{2}\right]+a_{1} \Delta \bar{b}_{2}-a_{2} \Delta \bar{b}_{1}, a=T_{1} a_{2}-T_{2} a_{1}, b=T_{2}{ }^{*} b_{1}-T_{1}^{*} b_{2}$, and the pairing $\Delta$ of $A$ and $\bar{A}$ into $H(A)$ is given by $a \Delta \bar{b}=L(a b)+[L(a)$, $L(b)]$ (see [1]).

Now let $M$ be an ideal of $A$. Let $j_{1}(M)=\{T \in H(A) \mid T A \sqsubseteq M\}$. $j_{1}(M)$ is an ideal in $H(A), j_{1}(M)$ contains the ideal $i(M)$ of $H(A)$ generated by the mappings $L(m), m \in M$. If we let $I(M)=i(M) \oplus M \oplus \bar{M}$ and $J_{1}(M)=j_{1}(M) \oplus M \oplus \bar{M}$, and note that $i(M)^{*} \subseteq i(M)$, then we see that $I(M)$ is an ideal in $\mathscr{L}(A)$. In this note, we shall give an example to show that $J_{1}(M)$ need not be an ideal in $\mathscr{L}(A)$. We first 
note some equivalent conditions for $J_{1}(M)$ to be an ideal of $\mathcal{L}(A)$.

Theorem. Let $A$ be a commutative admissible algebra, $M$ an ideal of $A$. Then the following three conditions are equivalent:

(1) $A / M$ is admissible

(2) $J_{1}(M)$ is an ideal of $\mathbb{L}(A)$

(3) $j_{1}(M)^{*} \subseteq j_{1}(M)$.

Proof. $(1) \Rightarrow(2)$. The natural homomorphism of $A$ onto $A / M$ induces a homomorphism of $\mathfrak{R}(A)$ onto $\mathfrak{R}(A / M)$, by replacing all vartables from $A$ which appear by their cosets modulo $M$. The kernel of this homomorphism has $M$ and $\bar{M}$ in the second and third components, respectively, and the first component is $j_{1}(M)$, i.e., the kernel is $J_{1}(M)$, an ideal of $\mathcal{Q}(A)$.

$(2) \Rightarrow(3)$. Let $T \in j_{1}(M), a \in A$. Then $\left.[0,0, \bar{a}),(T, 0, \overline{0})\right]=(0,0$, $\bar{b}) \in J_{1}(M)$, where $b=T^{*} a \in M$. Hence $T^{*} \in j_{1}(M)$.

$(3) \Rightarrow(1)$. We wish to show $H^{-}(A / M) \cap H^{+}(A / M)=0$. Let $a \rightarrow a^{\prime}$ be the natural homomorphism of $A$ onto $A / M$. Let $T^{\prime \prime}=$ $L\left(a^{\prime}\right)+\sum\left[\left[L\left(b_{i}^{\prime}\right), L\left(c_{i}^{\prime}\right)\right], L\left(d_{i}^{\prime}\right)\right]+\cdots=\sum\left[L\left(e_{j}^{\prime}\right), L\left(f_{j}\right)\right]+\cdots$ be in $H^{-}(A / M) \cap H^{+}(A / M)$. Then $U^{\prime}=L\left(a^{\prime}\right)-\sum\left[L\left(e_{j}^{\prime}\right), L\left(f_{j}^{\prime}\right)\right]+\sum\left[\left[L\left(b_{\imath}^{\prime}\right)\right.\right.$, $\left.\left.L\left(c_{i}^{\prime}\right)\right], L\left(d_{i}^{\prime}\right)\right]-\cdots=0, \quad$ so $\quad U=L(\alpha)-\sum\left[L\left(e_{j}\right), L\left(f_{j}\right)\right]+\sum\left[\left[L\left(b_{i}\right)\right.\right.$, $\left.\left.L\left(c_{i}\right)\right], L\left(d_{i}\right)\right]-\cdots$ is in $j_{1}(M)$. If we write $U=U^{-}+U^{+}, U^{-} \in H^{-}(A)$, $U^{+} \in U^{+}(A)$, then $U^{*}=U^{-}-U^{+} \in j_{1}(M)$, so $U^{-}, U^{+} \in j_{1}(M)$. But $U^{-}=$ $L(a)+\sum\left[\left[L\left(b_{i}\right), L\left(c_{i}\right)\right], L\left(d_{i}\right)\right]+\cdots$, so $T^{\prime}=0$. This proves the theorem. We remark that $(1) \Rightarrow(2)$ follows from $(2.8)$ of [1].

If $A$ is a commutative associative algebra, then $H(A)=L(A)$, so $A$ is admissible. Hence the conditions of the theorem hold for any ideal in a commutative associative algebra. If $A$ is a commutative Jordan algebra, then $L(A)$ is a Lie triple system, and $H(A)=$ $L(A)+[L(A), L(A)]$. If, in addition, $A$ has a unit element 1 , then $H(A)=L(A) \oplus[L(A), L(A)]$, so $A$ is admissible. Hence the conditions of the theorem hold for any ideal in a commutative Jordan algebra with unit element.

We now present an example of a commutative Jordan algebra without unit element, which is admissible, but which has an ideal $M$ which does not satisfy the conditions of the theorem. We consider three-by-three matrices over $F$, with $e_{i j}$ denoting the usual matrix units. Consider the four-dimensional Jordan algebra $A$ with basis $e=e_{11}+e_{33}, x=e_{12}, y=e_{23}, z=e_{13}$, and multiplication $a b+b a$ for $a$, $b \in A . \quad L(A)$ is four-dimensional, with basis $L(e), L(x), L(y), L(z)$. Let $T_{1}=[L(e), L(x)], T_{2}=[L(e), L(y)] . \quad T_{1}$ and $T_{2}$ form a basis for $[L(A)$, $L(A)]$. Let $\alpha L(e)+\beta L(x)+\gamma L(y)+\delta L(z)=\rho T_{1}+\tau T_{2}$. Applying to $e$ yields $\alpha=\delta=0, \rho=-\beta, \tau=-\gamma$. Applying to $x$ yields $\gamma=0$, and applying to $y$ yields $\beta=0$. Hence $L(A) \cap[L(A), L(A)]=0$. Since 
$A$ is a Jordan algebra, $H(A)=L(A) \oplus[L(A), L(A)]$, and $A$ is admissible.

Let $M=F z$, an ideal of $A$. Set $T=L(x)+T_{1}$ in $H(A) . T \in j_{1}(M)$ but $T^{*}=L(x)-T_{1}$ satisfies $T^{*}(e)=2 x \notin M$, so $j_{1}(M)^{*} \nsubseteq j_{1}(M)$. Noting the other conditions of the theorem, $A^{\prime}=A / M$ is not admissible as $L\left(x^{\prime}\right)=\left[L\left(x^{\prime}\right), L\left(e^{\prime}\right)\right] \neq 0$, and $J_{1}(M)$ is not an ideal in $\mathcal{L}(A)$, as $[(0,0$, $\bar{e}),(T, 0,0)]=(0,0,2 \bar{x}) \notin J_{1}(M)$.

COROLlary. The class of admissible algebras is not definable by polynomial identities.

This follows since our example shows that the class of admissible algebras is not closed under homomorphic images.

The algebra $A / M$ of the example is a commutative Jordan algebra which is not admissible. Another example would be the algebra with basis $e=e_{11}+e_{22}, x=e_{21}, y=e_{31}, z=e_{32}$, and multiplication $a b+b a$. Then $L(y)=[L(y) L(e)] \neq 0$.

In [1], an ideal $j(M)$ of $H(A)$ is introduced, $j(M)=\{T \in H(A) \mid T A \subseteq M$, $T^{*} A \subseteq M$. Then $j(M)^{*} \subseteq j(M)$ and $J(M)=j(M) \oplus M \oplus \bar{M}$ will be an ideal in $\mathfrak{L}(A)$. For commutative Jordan algebras with unit element, $j(M)=j_{1}(M)$, but our example shows that in general $j(M) \cong j_{1}(M)$, even for admissible Jordan algebras $A$. For an admissible commutative Jordan algebra $A, \mathcal{L}(A)$ is a Lie algebra. This is proved in [1] for Jordan algebras with unit element. If $A$ does not have a unit element, adjoin 1 to get the Jordan algebra $A_{1}$. The Lie algebra $\&\left(A_{1}\right)$ has a subalgebra $H_{A_{1}}(A) \oplus A \oplus \bar{A}$, where $H_{A_{1}}(A)$ is the enveloping Lie algebra of the multiplications in $A_{1}$ by elements of $A$. This subalgebra has a homomorphic image obtained by restricting the elements of $H_{A_{1}}(A)$ to $A$. But this image is $\mathscr{L}(A)$, so $\mathscr{L}(A)$ is a Lie algebra.

In conclusion, it might be interesting to find an example of a commutative admissible algebra $A$ with unit element with an ideal $M$ which fails to satisfy the conditions of the theorem. Such an $A$ could not be a Jordan algebra or an associative algebra.

\section{REFERENCE}

1. M. Koecher, Imbedding of Jordan algebras into lie algebras, I, Amer. J. Math. (1967), 787-816.

Received February 23, 1968. Research supported by National Science Foundation grant GP-7162. 



\section{PACIFIC JOURNAL OF MATHEMATICS}

\section{EDITORS}

H. ROYDEN

Stanford University

Stanford, California

Richard Pierce

University of Washington

Seattle, Washington 98105
J. DugundJI

Department of Mathematics

University of Southern California

Los Angeles, California 90007

BASIL GORDON

University of California

Los Angeles, California 90024

\section{ASSOCIATE EDITORS}

E. F. BECKENBACH

B. H. NeUmanN

F. WOLF

K. YOSHIDA

\section{SUPPORTING INSTITUTIONS}

UNIVERSITY OF BRITISH COLUMBIA

CALIFORNIA INSTITUTE OF TECHNOLOGY

UNIVERSITY OF CALIFORNIA

MONTANA STATE UNIVERSITY

UNIVERSITY OF NEVADA

NEW MEXICO STATE UNIVERSITY

OREGON STATE UNIVERSITY

UNIVERSITY OF OREGON

OSAKA UNIVERSITY

UNIVERSITY OF SOUTHERN CALIFORNIA
STANFORD UNIVERSITY

UNIVERSITY OF TOKYO

UNIVERSITY OF UTAH

WASHINGTON STATE UNIVERSITY

UNIVERSITY OF WASHINGTON

$* * \quad * \quad *$
AMERICAN MATHEMATICAL SOCIETY
CHEVRON RESEARCH CORPORATION
TRW SYSTEMS
NAVAL WEAPONS CENTER




\section{Pacific Journal of Mathematics}

Vol. 30, No. $1 \quad$ September, 1969

William Wells Adams, Simultaneous diophantine approximations and cubic irrationals ..................................... 1

Heinz Bauer and Herbert Stanley Bear, Jr., The part metric in convex

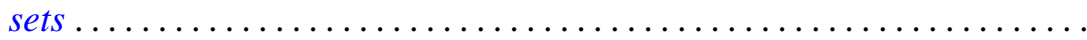

L. Carlitz, A note on exponential sums ...................... 35

Vasily Cateforis, On regular self-injective rings ................. 39

Franz Harpain and Maurice Sion, A representation theorem for measures on infinite dimensional spaces ......................... 47

Richard Earl Hodel, Sum theorems for topological spaces .............. 59

Carl Groos Jockusch, Jr. and Thomas Graham McLaughlin, Countable

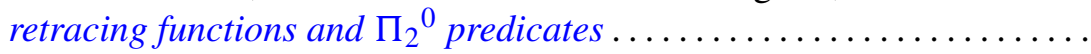

Bjarni Jónsson and George Stephen Monk, Representations of primary

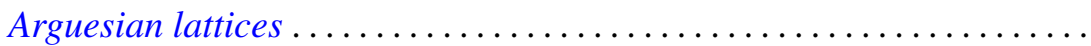

Virginia E. Walsh Knight, A continuous partial order for Peano continua...................................... 141

Kjeld Laursen, Ideal structure in generalized group algebras ........... 155

G. S. Monk, Desargues' law and the representation of primary lattices . . . 175

Hussain Sayid Nur, Singular perturbation of linear partial differential equation with constant coefficients ..........................

Richard Paul Osborne and J. L. Stern, Covering manifolds with cells ... 201

Keith Lowell Phillips and Mitchell Herbert Taibleson, Singular integrals in several variables over a local field...

James Reaves Smith, Local domains with topologically $T$-nilpotent radical....

Donald Platte Squier, Elliptic differential equations with discontinuous coefficients .................................

Tae-il Suh, Algebras formed by the Zorn vector matrix...

Earl J. Taft, Ideals in admissible algebras . .................... 259

Jun Tomiyama, On the tensor products of von Neumann algebras........ 263

David Bertram Wales, Uniqueness of the graph of a rank three group ..... 271

Charles Robert Warner and Robert James Whitley, A characterization of regular maximal ideals ......................... 